\title{
Original
}

\section{Dermatitis actínica crónica en el mundo laboral}

\author{
Chronic actinic dermatitis in laboral world
}

\section{María Teresa López Villaescusa ', Fabiana Robuschi Lestouquet ${ }^{2}$, Jaritzy Negrín González ${ }^{3}$ Roberto César Muñoz González ${ }^{4}$, Rubén Landa García ${ }^{5}$, Luis Conde- Salazar Gómez ${ }^{6}$.}

1. Servicio de Dermatología. Complejo Hospitalario Universitario de Albacete. España.

2. Servicio de Dermatología. Hospital Virgen de la Salud. Toledo. España.

3. Servicio de Alergología. Hospital Clínico San Carlos. Madrid.

4. Servicio Medicina Laboral. FREMAP. Unidad Docente. Comunidad de Castilla La Mancha. Toledo. España.

5. Servicio Medicina Laboral. ASEPEYO MATEPSS N 151 . Unidad Docente. Comunidad de Castilla La Mancha. Toledo. España.

6. Servicio de Dermatología Laboral. Escuela Nacional de Medicina del Trabajo. Instituto de Salud Carlos III. Madrid. España.

\section{Correspondencia:}

María Teresa López Villaescusa

Servicio de Dermatología

Complejo Hospitalario Universitario de Albacete

Hermanos Falcó n. ${ }^{\circ 37}$

02006 Albacete. España

Tfno: (+34) 661966448

E-mail: lopezvillaescusa@hotmail.com

\section{Resumen}

Introducción: Del espectro de radiación electromagnética la radiación solar es la principal causante de las respuestas fotobiológicas en la piel, como la dermatitis actínica crónica.

Objetivo: Realizamos un estudio retrospectivo de los pacientes diagnosticados de dermatitis actínica crónica desde 1987 hasta la actualidad, en el servicio de Dermatología Laboral del Instituto de Salud Carlos III, de Madrid.

Material y métodos: Se valoró edad, sexo, antecedentes personales, actividad laboral como factor predisponente, características de las lesiones y el resultados a las pruebas epicutáneas de alergia. No se realizó estudio fotobiológico.

Resultados: Se estudiaron 6 pacientes varones, con edades comprendidas entre los 39 y 56 años, con importante exposición solar laboral. La clínica era similar con afectación ezcematosa de zonas fotoexpuestas. El resultado de las pruebas epicutáneas fue positivo en dos pacientes.

Discusión: El 75\% de los casos de dermatitis actínica crónica, se asocia con un alérgeno. Las principales limitaciones de los casos son la falta de estudio fotobiológico en los pacientes. El trabajo profesional al aire 
libre lleva implícito riesgos primarios, de tipo físico, como la exposición a radiación ultravioleta, que es necesario controlar para lograr una mayor seguridad en el trabajo.

Med Segur Trab (Internet) 2012; 59 (227) 128-135

Palabras clave: Alérgeno, dermatitis actínica crónica, riesgo laboral, fotoprotección, radiación ultravioleta.

Abstract

Introduction: In the spectrum of electromagnetic radiation, solar radiation is the main cause of photobiological responses in the skin, such as chronic actinic dermatitis.

Objetive: We performed a retrospective study of patients, with chronic actinic dermatitis seen in our department:"Dermatología Laboral del Instituto de Salud Carlos III, Madrid", from 1987 to present.

Material and methods: We evaluated age, sex, personal history, work activity as a predisposing factor, characteristic of lesions and the results of allergy patch tests. Photobiological study was not performed.

Results: We studied 6 male patients, aged between 39 and 56, with significant sun exposure at work. The clinic was similar, with eczematous lesions in photoexposed areas. The result of patch testing was positive in two patients.

Discussion: 75 per cent of cases of chronic actinic dermatitis are associated with an allergen. The main limitations are the lack of photobiological study in patients. The outdoor professional work implies primary risk, such as ultraviolet radiation exposition, which is necessary to control for greater safety al work.

Med Segur Trab (Internet) 2012; 59 (227) 128-135

Key words: Allergen, chronic actinic dermatitis, laboral risk, photoprotection, ultraviolet radiation. 


\section{INTRODUCCIÓN}

La radiación electromagnética, es una forma de energía, que se puede describir según su frecuencia (número de oscilaciones por segundo), su longitud de onda (distancia alcanzada por oscilación) o por cuantos de energía (fotones). La radiación electromagnética forma un espectro continuo de energía que abarca unas longitudes de onda desde fracciones de nanómetros hasta miles de metros, a su vez, la radiación solar, es el conjunto de radiaciones electromagnéticas emitidas por el sol. De este espectro, la radiación solar se constituye por la radiación ultravioleta y la radiación visible, que son las principales causantes de las respuestas fotobiológicas en la piel.

La radiación ultravioleta se separa en tres subdivisiones, la radiación UVC, comprende un rango de longitud de onda de 200 a $290 \mathrm{~nm}$, que es absorbida por la capa de ozono de la estratosfera. El rango que va desde los 290 a $320 \mathrm{~nm}$, es la radiación UVB, también conocido como espectro de la quemadura solar. La radiación UVA, va desde los 320 a $400 \mathrm{~nm}$, por último, el espectro de la luz visible comprende desde los 400 a $760 \mathrm{~nm}$. A mayor longitud de onda mayor penetración pero menor energía $^{1}$.

La parte de los rayos UV, que alcanza la piel, es absorbida por biomoléculas, provocando así, respuestas fotoquímicas y fotobiológicas. Entre sus efectos beneficiosos, la exposición solar es importante para la síntesis de vitamina D y ajuste de relojes internos. Desde el punto de vista negativo, produce efectos perjudiciales agudos y crónicos como quemaduras, fotoenvejecimiento y cáncer de piel. Por otro lado, solo determinadas personas desarrollan reacciones anómalas, también conocidas como fotodermatosis, entre ellas, las dermatosis fotoagravadas, por ejemplo lupus, enfermedad de Darier, porfirias, etc, las respuestas fototóxicas o fotoalérgicas, las enfermedades por defecto de reparación del ADN y las fotodermatosis idiopáticas. En este grupo, se ha comprobado que la respuesta inmunológica anormal en el huésped, juega un papel importante en la etiopatogenia de la enfermedad ${ }^{2}$.

La dermatitis actínica crónica, enfermedad que se incluye dentro del grupo de las fotodermatosis idiopáticas, es un término introducido en 1979 por Hawk y Magnus para unificar conceptos. Por tanto se incluye bajo este término los anteriormente conocidos como reticuloide actínico, reactividad a la luz persistente y ezcema fotosensible ${ }^{3}$.

Existen dos hipótesis que explican el mecanismo etiopatogénico. En la primera de ellas, un constituyente de la piel normal se altera y se vuelve antigénico, tras la unión fotoquímica covalente a un hapteno, dependiendo de UVA principalmente y en menor medida de UVB, durante una reacción fotoalérgica local. Una vez inducida la respuesta local, se perpetúa la respuesta inflamatoria, sin necesidad de que persista la reacción fotoalérgica previa ${ }^{4}$. Esta teoría explica la presencia de un fotoalérgeno, como desencadenante del cuadro. En los estudios descritos, los principales fotoalérgenos relacionados son: plantas de la familia Compositae, fragancias, breas, caucho, pantallas solares, fármacos como la fenotiacidas y metales como el dicromato potásico ${ }^{5,6,7}$.

La segunda teoría, se basa en una reacción fototóxica, con daño directo sobre el DNA ${ }^{8}$. De este modo, se explica la presencia de dermatitis actínica crónica, sin necesidad de fotoalergia previa, representando el estado final de trastornos predisponentes como, dermatitis alérgica de contacto, ezcemas endógenos o incluso, aparición de novo en sujetos sanos?.

La dermatitis actínica crónica se suele presentar en varones alrededor de los 50 años de edad. Comienza con una erupción en zonas fotoexpuestas como cara, zona 
de escote y dorso de manos, tras exposición al sol, respetando la profundidad de los pliegues de la piel. Al comienzo las lesiones suelen ser de tipo eczematoso, y posteriormente evolucionan a placas eritematosas, brillantes e infiltradas, que hacen que el rostro adopte un aspecto leonino, con marcado engrosamiento de la piel. Aparece alteración de la pigmentación de distribución irregular, acompañado de prurito de difícil control ${ }^{10}$. Ocasionalmente puede extenderse a zonas adyacentes no fotoextuestas, e incluso provocar eritrodermia, difícil de distinguir de un linfoma cutáneo de células $\mathrm{T}$, tipo micosis fungoide, lo que ha contribuido a encuadrarlo en el diagnóstico genérico de pseudolinfoma ${ }^{11,12}$.

De hecho, la histopatología, en fases iniciales presenta, un patrón característico de ezcema agudo o subagudo con espongiosis epidérmica, acantosis, y en ocasiones hiperplasia, junto con infiltrado celular linfocítico, en mayor medida perivascular, confinado a la dermis superior. Sin embargo, en fases más avanzadas se puede encontrar, una histología similar al linfoma cutáneo de células $\mathrm{T}$, con microabscesos de tipo Pautrier epidérmicos y un infiltrado mononuclear epidermotropo intenso. No se ha observado clonalidad de linfocitos $\mathrm{T}$ y predominio de linfocitos $\mathrm{T}$ supresores sobre los colaboradores, a diferencia de la micosis fungoide ${ }^{13,14}$.

Existen unos criterios básicos para diagnosticar una dermatitis actínica crónica $^{15}$.

1. La presencia de pápulas o placas en piel fotoexpuesta, a veces con extensión zonas no fotoexpuestas, o bien una eritrodermia generalizada.

2. El fototest revela marcada fotosensibilidad, caracterizada por la reducción de Dosis Eritematosa Mínima (DEM) a radiación UVA, UVB y luz visible.

3. Infiltrado de linfocitos en dermis, algunos de ellos con características atípicas.

En cuanto al tratamiento, es imprescindible una fotoprotección eficaz, y evitar la exposición a alérgenos responsables en el caso de que éstos se hayan detectado. Se puede emplear corticoide por vía tópica o sistémica. También se emplean inmunosupresores, en casos resistentes como azatioprina o ciclosporina, Otros tratamientos son la cloroquina y la PUVA-terapia, con resultados parciales. Su curso es crónico con brotes recurrentes que le condicionan una enfermedad grave e incapacitante ${ }^{16}$.

\section{OBJETIVO}

En este artículo, presentamos los casos vistos en el servicio de Dermatología Laboral del Instituto de Salud Carlos III, de Madrid. Se realiza un estudio retrospectivo de los pacientes diagnosticados de dermatitis actínica crónica desde 1987 hasta la actualidad.

\section{MATERIAL Y MÉTODOS}

A través de la historia clínica se valoró edad, sexo, antecedentes personales, factores predisponentes para padecer dermatitis actínica crónica, con especial hincapié en la actividad laboral, características de las lesiones y el resultados a las pruebas epicutáneas de alergia realizadas. Por motivos técnicos, no se pudo realizar estudio fotobiológico a los pacientes, solamente se realizó a uno de ellos en otro centro, observándose fotosensibilidad marcada para UVA, UVB y luz visible con el fototest, y negatividad para el fotoparche. 


\section{RESULTADOS}

En la tabla 1, se expone respectivamente los hallazgos epidemiológicos, y clínicos. El resultado obtenido fueron 6 pacientes, todos ellos varones, con edades comprendidas entre los 39 y 56 años, en el momento del diagnóstico. La actividad laboral con importante exposición a radiación solar fue el factor predisponente para desarrollo de dermatitis actínica crónica en todos los pacientes. Esta actividad laboral incluía trabajadores de la construcción o conductores con largas horas de exposición solar. En la forma de afectación clínica, destacamos la constante afectación de zonas fotoexpuestas, como la cara, zona de escote y dorso de manos, en todos los pacientes. Ninguno tenía afectación de áreas no expuestas. Las lesiones se caracterizaban por ser pápulas que confluían formando placas eritematodescamativas, infiltradas, liquenificadas, con bordes bien diferenciados, delimitado a las zonas fotoexpuestas. Respetaban pliegues retroauriculares e interdigitales en la afectación de dorso de manos.

Tabla 1. Hallazgos epidemiológicos y clínicos de los pacientes atendidos en el servicio de Dermatología Laboral del Instituto de Salud Carlos III, Madrid, desde 1987 hasta la actualidad

\begin{tabular}{|c|c|c|c|c|c|c|c|}
\hline Sexo & Edad & $\begin{array}{l}\text { Antecedentes } \\
\text { personales }\end{array}$ & Profesión & $\begin{array}{c}\text { Localización } \\
\text { de lesiones }\end{array}$ & $\begin{array}{l}\text { Tiempo } \\
\text { de } \\
\text { evolución }\end{array}$ & $\begin{array}{c}\text { Batería pruebas } \\
\text { epicutáneas }\end{array}$ & Resultados \\
\hline Varón & 51 & $\begin{array}{l}\text { Toma de } \\
\text { antibiótico } \\
\text { (quinolona) } \\
\text { por infección } \\
\text { de vías } \\
\text { respiratorias } \\
\text { bajas. }\end{array}$ & Conductor & $\begin{array}{l}\text { Cara y } \\
\text { zona de } \\
\text { escote }\end{array}$ & $\begin{array}{c}4 \\
\text { meses }\end{array}$ & $\begin{array}{l}\text { - Estándar } \\
\text { - Cosméticos } \\
\text { - Fragancias } \\
\text { - Sustancias } \\
\text { propias } \\
\text { de higiene } \\
\text { personal }\end{array}$ & Negativo \\
\hline Varón & 45 & Sin interés & Construcción & $\begin{array}{l}\text { Dorso de } \\
\text { manos }\end{array}$ & 1 año & $\begin{array}{l}\text { - Estándar } \\
\text { - Gomas }\end{array}$ & Negativo \\
\hline Varón & 56 & Sin interés & Construcción & $\begin{array}{l}\text { Cara y } \\
\text { zona de } \\
\text { escote }\end{array}$ & $\begin{array}{c}7 \\
\text { meses }\end{array}$ & $\begin{array}{l}\text { - Estándar } \\
\text { - Construcción }\end{array}$ & Negativo \\
\hline Varón & 39 & Sin interés & Hostelería & $\begin{array}{l}\text { Cara, } \\
\text { zona de } \\
\text { escote y } \\
\text { dorso de } \\
\text { manos }\end{array}$ & $\begin{array}{c}4 \\
\text { meses }\end{array}$ & - Estándar & Negativo \\
\hline Varón & 52 & Sin interés & Construcción & $\begin{array}{l}\text { Cara y } \\
\text { zona de } \\
\text { escote }\end{array}$ & $\begin{array}{c}10 \\
\text { meses }\end{array}$ & $\begin{array}{l}\text { - Estándar } \\
\text { - Construcción }\end{array}$ & $\begin{array}{l}\text { Positividad } \\
\text { - Dicromato } \\
\text { potásico } \\
\text { - Cloruro de } \\
\text { cobalto }\end{array}$ \\
\hline Varón & 54 & Sin interés & Construcción & $\begin{array}{l}\text { Cara y } \\
\text { zona de } \\
\text { escote }\end{array}$ & 2 años & - Estándar & $\begin{array}{l}\text { Positividad } \\
\text { - Dicromato } \\
\text { potásico } \\
\text { - Resina de } \\
\text { butilformal- } \\
\text { dehido }\end{array}$ \\
\hline
\end{tabular}


Se realizaron pruebas alérgicas de contacto, con la batería estándar para pruebas epicutáneas del Grupo Español de investigación para la Dermatitis de Contacto (GEIDC), también se incluyó baterías específicas de acuerdo a la situación personal-laboral de cada paciente. El resultado obtenido fue negativo en cuatro pacientes, en el resto de pacientes se observaba positividad para alérgenos; en uno de ellos para dicromato potásico y cloruro de cobalto y en otro se objetivó positividad para dicromato potásico y resina de butilformaldehído.

Se realizó estudio histopatológico en todos los pacientes que mostró grados variables de espongiosis, acantosis e infiltrado linfocitario perivascular en dermis.

\section{DISCUSIÓN}

De acuerdo con los casos presentados y revisando en la literatura las series y casos descritos de dermatitis actínica crónica llama la atención, que en el 75\% de los casos existe asociación con un alérgeno. Las principales limitaciones de los casos son la falta de estudio fotobiológico en los pacientes.

Las dermatosis producidas por afectación solar siempre han sido procesos poco estudiados, debido en muchas ocasiones a que el propio enfermo conocía la causa y no consultaba. Además de ello, cuando el paciente consultaba, el médico banalizaba la entidad, otorgando una infravaloración al cuadro, o no se disponía del material adecuado para la realización de un estudio fotobiológico, que indicara la causa del problema dermatológico.

Pero el problema es más complejo, cuando influye el ámbito laboral, ya que en este caso un diagnóstico certero puede ayudar a declarar una enfermedad desconocida.

Un sector no despreciable de la población laboral se encuentra expuesto a radiación ultravioleta, entre ellos: empleados de la construcción, jardineros, conductores, agrícolas, ganaderos, pescadores, trabajadores en áreas deportivas, guías turísticos, etc. En todos ellos, la exposición suele ser continua y de larga duración, por ello, la mejor forma de controlar el riesgo, es mediante actuaciones que quedan englobadas en dos grupos ${ }^{17}$ :

1. Prevención primaria, cuya finalidad es disminuir la incidencia evitando o reduciendo las causas de la enfermedad. La prevención primaria basa su actuación en tratar de modificar los hábitos y conductas erróneas: incrementar el conocimiento de la población respecto a la enfermedad y la exposición a radiación ultravioleta, el principal factor desencadenante de la enfermedad (uso de cremas fotoprotectoras, insistiendo en su correcta aplicación, cambio en los hábitos de exposición solar y utilización de ropa adecuada).

2. Prevención secundaria, que consiste en la detección temprana de las lesiones, una vez que ya han aparecido.

3. El trabajo profesional al aire libre lleva implícito riesgos primarios, de tipo físico, como la exposición a radiación ultravioleta, que es necesario controlar para lograr una mayor seguridad en el trabajo.

Figura 1. Caso 1. Lesiones infiltradas que dan al rostro un aspecto leonino con engrosamiento de la piel

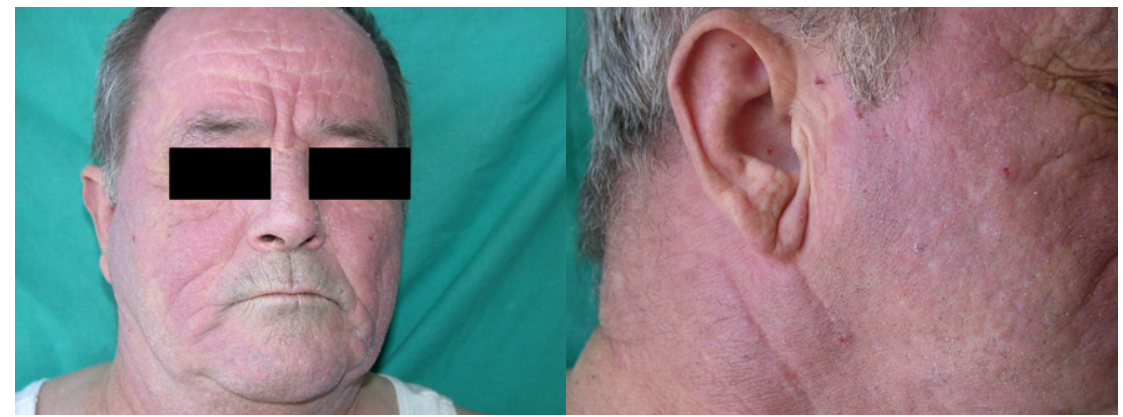


Figura 2. Caso 2. Placas etriemato-descamativas, infiltradas, que afectan a la cara

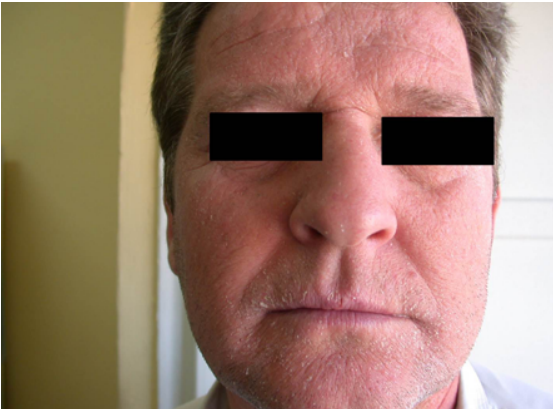

Figura 3. Caso 3. Afectación de la zona fotoexpuesta. A mayor aumento, respeto del pliegue retroauricular

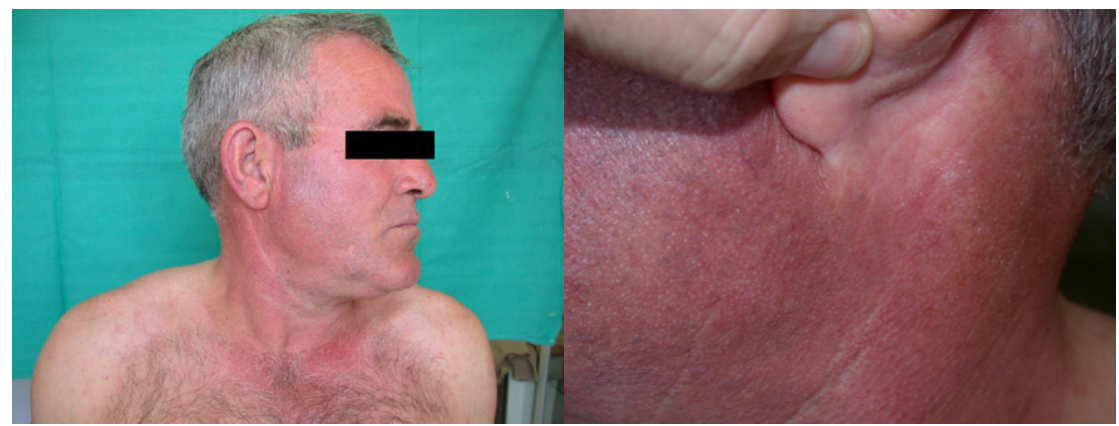

Figura 4. Caso 4. Pápulas coalesciendo formando placas en el dorso de la mano

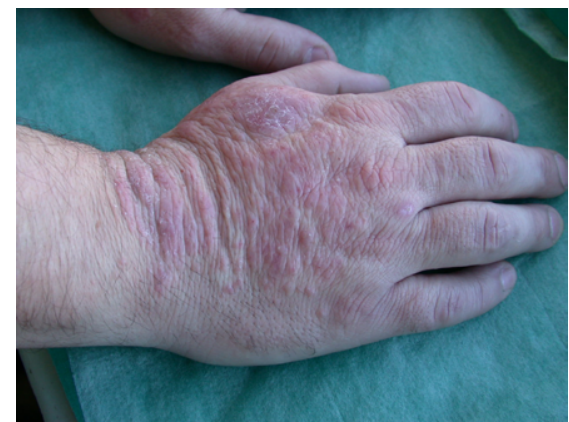

Figura 5. Caso 5. Delimitación bien definida de las lesiones en zonas fotoexpuestas

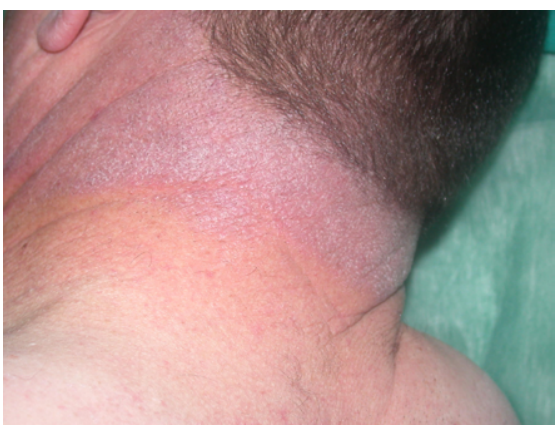




\section{REFERENCIAS BIBLIOGRÁFICAS}

1. Smith E, Kiss F, Porter RM, Anstey AV. A review of UVA-mediated photosensitivity disorders.. Photochem Photobiol Sci. 2012; 11(1):199-206.

2. Luy H, Frenk E, Applegate LA. Ultraviolet A-induced cellular membrane damage in the photosensitivity dermatitis/actinic reticuloid syndrome. Photodermatol Photoimmunol Photomed. 1994; 10(3):126-33.

3. Dawe RS, Crombie IK, Ferguson J. The natural history of chronic actinic dermatitis.. Arch Dermatol. 2000; 136(10):1215-20.

4. Zak-Prelich M, Schwartz RA. Actinic reticuloid. Int J Dermatol. 1999; 38(5):335-42.

5. Dawe RS, Green CM, MacLeod TM, Ferguson J. Daisy, dandelion and thistle contact allergy in the photosensitivity dermatitis and actinic reticuloid syndrome. Contact Dermatitis. 1996; 35(2):109-10.

6. Hannuksela M. Skin contact reactions caused by plants. Duodecim. 2009; 125(13):1398-406.

7. Bilsland D, Ferguson J. Contact allergy to sunscreen chemicals in photosensitivity dermatitis/actinic reticuloid syndrome (PD/AR) and polymorphic light eruption (PLE). Contact Dermatitis. 1993; 29(2):70-3.

8. Healy E, Rogers S. Photosensitivity dermatitis/actinic reticuloid syndrome in an Irish population: a review and some unusual features. Acta Derm Venereol. 1995; 75(1):72-4.

9. Russell SC, Dawe RS, Collins P, Man I, Ferguson J. The photosensitivity dermatitis and actinic reticuloid syndrome (chronic actinic dermatitis) occurring in seven young atopic dermatitis patients. Br J Dermatol. 1998; 138(3):496-501.

10. Ravić-Nikolić A, Miličić V, Ristić G, Jovović-Dagović B, Mitrović S. Actinic reticuloid presented as facies leonine. Int J Dermatol. 2012; 51(2):234-6.

11. De Silva BD, McLaren K, Kavanagh GM. Photosensitive mycosis fungoides or actinic reticuloid?. Br J Dermatol. 2000; 142(6):1221-7.

12. Ploysangam T, Breneman DL, Mutasim DF. Cutaneous pseudolymphomas.. J Am Acad Dermatol. 1998; 38(6 Pt 1):877-95.

13. Bakels V, van Oostveen JW, Preesman AH, Meijer CJ, Willemze R. Differentiation between actinic reticuloid and cutaneous $\mathrm{T}$ cell lymphoma by $\mathrm{T}$ cell receptor gamma gene rearrangement analysis and immunophenotyping. J Clin Pathol. 1998; 51(2):154-8.

14. Bilsland D, Crombie IK, Ferguson J. The photosensitivity dermatitis and actinic reticuloid syndrome: no association with lymphoreticular malignancy. Br J Dermatol. 1994; 131(2):209-14.

15. Dawe RS, Ferguson J. Diagnosis and treatment of chronic actinic dermatitis. Dermatol Ther. 2003;16(1):4551.

16. Paquet P, Piérard GE. Severe chronic actinic dermatitis treated with cyclosporine: 2 cases. Ann Dermatol Venereol. 2001; 128(1):42-5.

17. Sánchez Conejo-Mir J. Campañas de prevención del melanoma. Piel. 2002; 17(10):457-65.

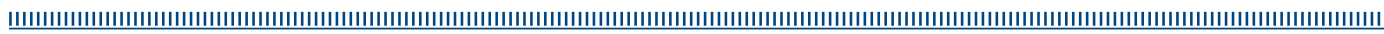

